\title{
Chirality-Dependent Amino Acid Modulation of RNA Folding
}

David A. Nicholson ${ }^{1,2}$ Abhigyan Sengupta, ${ }^{3}$ and David J. Nesbitt ${ }^{1,2,4}$

\footnotetext{
${ }^{1}$ JILA, National Institute of Standards and Technology and University of Colorado, Boulder, Colorado 80309 USA

${ }^{2}$ Department of Chemistry, University of Colorado, Boulder, Colorado 80309

${ }^{3}$ Department of Physics, Technical University of Munich, Garching b. Munich, Germany 85748

${ }^{4}$ Department of Physics, University of Colorado, Boulder, Colorado 80309 USA
}

\section{Supporting Information}



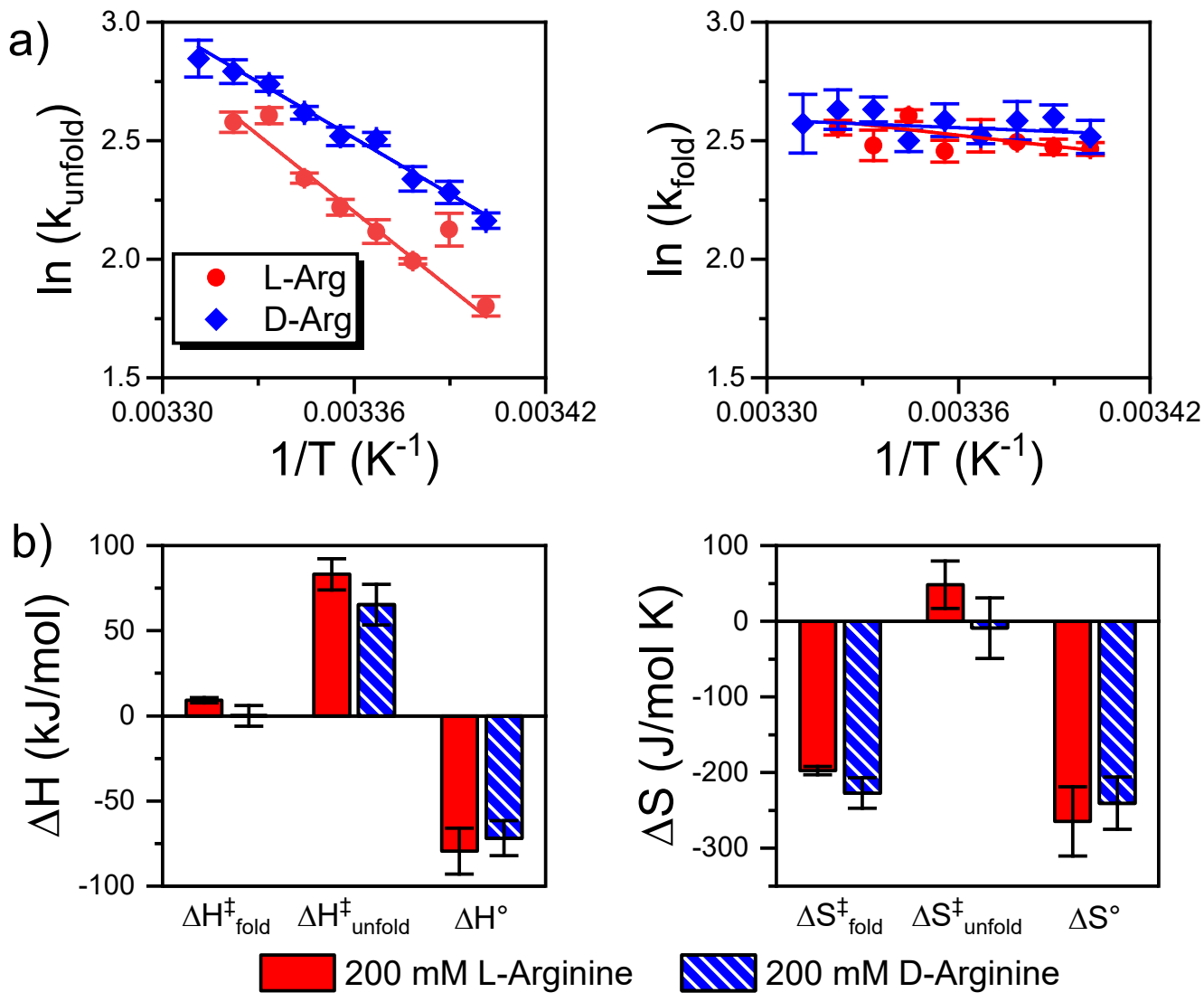

Figure S1. Temperature-dependent experiments for $200 \mathrm{mM} \mathrm{L}$ - and D-arginine. (a) Arrhenius plots for unfolding (left) and folding (right) rate constants. (b) Enthalpies $(\Delta \mathrm{H})$ and entropies $(\Delta \mathrm{S})$ parameters from linear fits. Both enantiomers of arginine yield the same fit results, within error. 


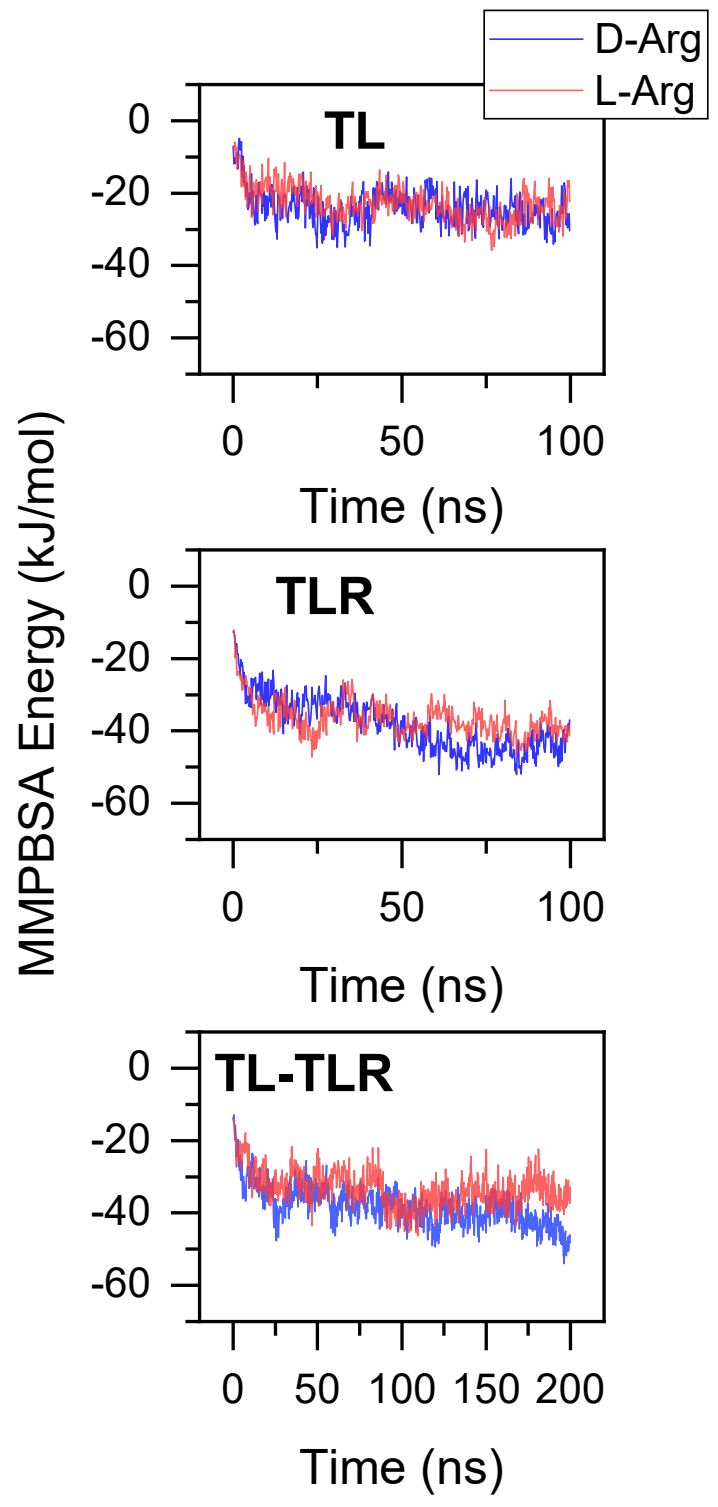

Figure S2. MMPBSA energies of arginine binding to RNA computed frame-by-frame for MD trajectories. Binding is with GAAA tetraloop (TL, top), 11-nt tetraloop receptor (TLR, middle), and docked tetraloop-tetraloop receptor (TL-TLR, bottom). 


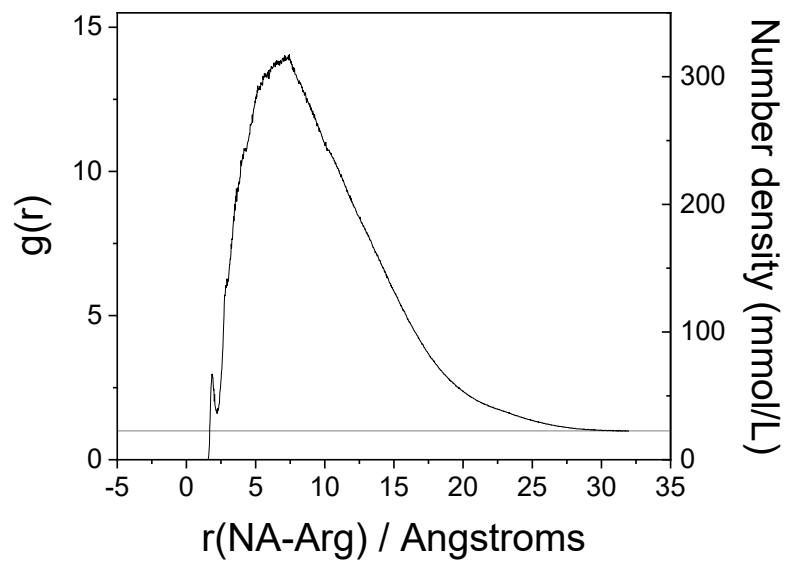

Figure S3. Representative radial distribution function $g(r)$ of the distance between nucleic acid and arginine heavy atoms for the TL system. Large enrichment at medium distances is observed, corresponding to arginine cation recruitment into the ion atmosphere of the nucleic acid. For other systems, similar local concentrations of 10-25x the bulk concentration are observed. Asymptotic values of the number density (right axis) are used to compute the effective bulk concentration. 
$\underline{\text { Movies }}$

1 - TL_Arginine.gif

2 - TLR_Arginine.gif

3 - TL-TLR_Arginine.gif

4 - TL_Lysine.gif

5 - TLR_Lysine.gif

6 - TL-TLR_Lysine.gif

Movies show 360-degree perspective of simulated MD systems with overlay of binding free energy isosurfaces at $\Delta \mathrm{G}=5 \mathrm{k}_{\mathrm{B}} \mathrm{T}$ (solid) and $\Delta \mathrm{G}=4 \mathrm{k}_{\mathrm{B}} \mathrm{T}$ (transparent) for binding of arginine (1-3) and lysine (4-6) for the L-enantiomer (left, red) and the D-enantiomer (right, blue). 